\title{
Presymptomatic treatment of classic late-infantile neuronal ceroid lipofuscinosis with cerliponase alfa
}

\author{
J. Schaefers' ${ }^{1}$, L. J. van der Giessen², C. Klees ${ }^{3}$, E. H. Jacobs', S. Sieverdink' ${ }^{4}$ M. H. G. Dremmen ${ }^{5}$, J. K. H. Spoor ${ }^{6}$, \\ A. T. van der Ploeg ${ }^{1}$, J. M. P. van den Hout $^{1}$ and H. H. Huidekoper ${ }^{1 *}$ (D)
}

\begin{abstract}
Background: Neuronal ceroid lipofuscinosis type 2 (CLN2 disease) is a rare rapidly progressive neurodegenerative disorder, resulting in early death. Intracerebroventricular enzyme replacement therapy (ERT) with cerliponase alfa is now available and has shown to delay disease progression in symptomatic patients. It is yet unknown if cerliponase alfa can prevent disease onset in presymptomatic patients.
\end{abstract}

Results: We evaluated the effect of 2 years of intracerebroventricular ERT in two siblings with CLN2 disease, one symptomatic (age 47 months) and one presymptomatic (age 23 months) at treatment start, using the CLN2 Clinical Rating Scale (CLN2 CRS), Gross Motor Function Measure-66 (GMFM-66) for motor function, Bayley Scales of Infant and Toddler Development, 3rd Edition, Dutch (BSID-III-NL) for neurocognitive development, brain MRI, and visual evoked potentials (VEP), electroretinogram (ERG) and retinoscopy for visual function. On the CLN2 CRS patient 1 showed a decline from 3 to 2 in the combined motor and language score due to regression in language use (CLN2 CRS total score after 2 years of treatment: 8), whereas a decline of 2 or more points in the combined motor and language score would be expected without treatment. Patient 2 retained the maximum score of 3 in all 4 subdomains (CLN2 CRS total score after 2 years of treatment: 12). The GMFM-66 total score declined from 46 to 39 in patient 1 and showed an age-appropriate increase from 66 to 84 in patient 2. Cognitive-developmental age decreased from 24 to 11 months in patient 1, whereas an increase in cognitive-developmental age from 21 to 39 months was seen in patient 2. Cerebral and cerebellar atrophy observed on MRI in patient 1 at age 42 months (before treatment) was not observed in patient 2 at age 48 months (after 2 years of treatment).

Conclusion: We show that cerliponase alfa is able to delay the onset of symptoms when treatment is started in a presymptomatic stage of CLN2 disease. Our results advocate the start of treatment at an early age before symptom onset, but should be confirmed in a larger cohort study.

Keywords: Late-infantile neuronal ceroid lipofuscinosis, CLN2 disease, Tripeptidyl peptidase, Presymptomatic, Cerliponase alfa, Enzyme replacement therapy, Intracerebroventricular, Lysosomal storage disorder

\footnotetext{
*Correspondence: h.huidekoper@erasmusmc.nl

${ }^{1}$ Department of Pediatrics, Center for Lysosomal and Metabolic Diseases,

Erasmus MC, University Medical Center Rotterdam, PO Box 2040, 3000

CA Rotterdam, The Netherlands

Full list of author information is available at the end of the article
}

\begin{abstract}
Background
Neuronal ceroid lipofuscinosis type 2 (CLN2 disease; OMIM\#204500) is an autosomal recessive disease, caused by deficiency of the lysosomal enzyme tripeptidyl peptidase (TPP1; EC 3.4.14.9) [1,2]. The natural substrate and function of TPP1 are unknown, but its deficiency leads to the accumulation of auto fluorescent storage material
\end{abstract} original author(s) and the source, provide a link to the Creative Commons licence, and indicate if changes were made. The images or other third party material in this article are included in the article's Creative Commons licence, unless indicated otherwise in a credit line to the material. If material is not included in the article's Creative Commons licence and your intended use is not permitted by statutory regulation or exceeds the permitted use, you will need to obtain permission directly from the copyright holder. To view a copy of this licence, visit http://creativecommons.org/licenses/by/4.0/. The Creative Commons Public Domain Dedication waiver (http://creativeco mmons.org/publicdomain/zero/1.0/) applies to the data made available in this article, unless otherwise stated in a credit line to the data. 
(ceroid lipofuscin) in the lysosomes and neuronal loss [3]. In the classic late-infantile form of CLN2 disease this results in a rapidly progressive neurodegenerative disorder with a highly homogeneous course of disease. Typically, patients present between the age of 2 and 4 years with an unprovoked seizure, often a language delay will already be present. Thereafter, patients exhibit a rapid decline in cognitive, language, motor and visual function, with complete loss of motor and speech functions by $6-7$ years of age, followed by blindness and premature death between 8 and 13 years [4-7]. More than half of the patients are either homozygous or compound heterozygous for the splicing mutation c.509-1G $>$ A and/or the nonsense mutation c. $622 \mathrm{C}>\mathrm{T}[4,5,8,9]$. Progression and onset of disease is largely homogeneous and predictable for several genotypes [10-12].

Until 2017, only supportive treatment with a multidisciplinary approach was available for patients with CLN2 disease [13, 14]. However in 2017, the first intracerebroventricular enzyme replacement therapy with cerliponase alfa, a recombinant human TPP1 enzyme, was approved based on the results of the pivotal trial conducted in 23 CLN2 patients $[15,16]$. It was shown that patients treated with cerliponase alfa had a slower decline in the adjusted motor-language score of the CLN2 clinical rating scale, the primary outcome measure of the trial, compared to historical controls $(0.38 \pm 0,10$ vs $2.06 \pm 0.15$ points) after 48 weeks of treatment [15]. Although these results are very promising, long term efficacy has yet to be demonstrated. In addition, the potential of delaying disease onset when treatment is started in a presymptomatic stage of the disease remains to be shown and is currently being investigated in a phase 2 trial (NCT02678689).

In this paper, we report on the clinical outcome in two siblings with classic late-infantile CLN2 disease treated with cerliponase alfa for 2 years. The youngest sibling was started on treatment before the onset of clinical symptoms.

\section{Results \\ Case descriptions before initiation of treatment \\ Patient 1}

The index patient was the eldest daughter of non-consanguineous Dutch parents. She was born at term after an uncomplicated pregnancy and delivery. Her motor development was delayed, related to hip dysplasia which was treated with a Pavlik harness after which she became a bottom-shuffler and started walking at age 26 months. At age 30 months she was able to run and make knob puzzles. She spoke two-word sentences at age 24 months, and was able to speak three-word sentences and name objects at age 30 months. From age 39 months onward her parents started to notice the development of a tremor in her right arm and leg, and a loss in vocabulary. In the months thereafter she developed hypertonia of her legs, eventually leading to loss of the ability to stand and walk without support at the age of 43 months, the inability to produce two-word sentences or name objects at age 48 months, and eating difficulties.

Her first tonic-clonic seizure occurred at age 42 months, which was treated with levatiracetam. In retrospect her parents observed frequent absence seizures prior to this. They also noticed a change in behavior. She had always been a happy child, but at age 40 months she became more irritable, cried often and seemed frustrated. She also developed a sleeping disorder where she woke up the middle of the night and remained awake thereafter.

Whole exome sequencing was performed at the age of 44 months. This revealed the presence of the two most prevalent pathogenic variants in TPP1, c.622C > T; (p.Arg208*) and c.509-1G > C. Tripeptidyl peptidase deficiency was confirmed in leucocytes (TPP1 activity was $20.3 \mathrm{nmol} / \mathrm{h} / \mathrm{mg}$; control range $125-340 \mathrm{nmol} / \mathrm{h} / \mathrm{mg}$ ). At age 47 months treatment with cerliponase alfa was started at the Center for Lysosomal and Metabolic Diseases, Erasmus MC University Medical Center.

\section{Patient 2}

This patient is the younger sibling of patient 1 . He was born at term after an uncomplicated pregnancy and delivery. He started walking at age 14 months and was able to run and climb the stairs soon thereafter. His fine motor skills were age appropriate. He started using single words before his first birthday and he spoke twoword sentences, with a normal vocabulary and good non-verbal communication skills at age 21 months. He was diagnosed with CLN2 disease based on the diagnosis of his sister: he had the same TPP1 genotype and tripeptidyl peptidase deficiency in leucocytes was also confirmed (TPP1 activity: $16.9 \mathrm{nmol} / \mathrm{h} / \mathrm{mg}$; control range $125-340 \mathrm{nmol} / \mathrm{h} / \mathrm{mg}$ ). He did not exhibit any signs of epilepsy. He was started on treatment with cerliponase alfa at age 23 months. At that time there were no symptoms of CLN2 disease.

\section{Assessment of treatment efficacy CLN2 clinical rating scale (CLN2 CRS)}

At baseline (age 47 months) patient 1 had a score of 3 on the combined motor and language scale and a CLN2 CRS total score of 7 . After 15 months of treatment she lost 1 point on the language domain (CLN2 CRS combined motor-language score: 2 ). The score for her epilepsy fluctuated over time between 0 and 3, resulting in a CLN2 CRS total score fluctuating between 6 and 9 (Fig. 1). 


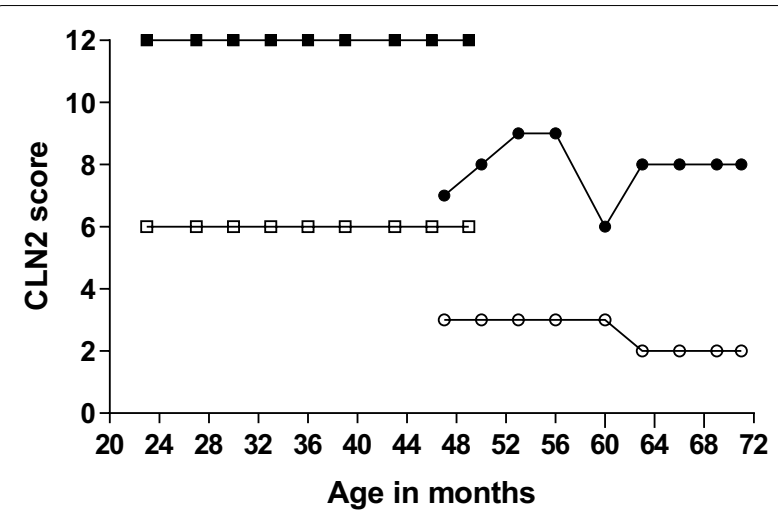

Fig. 1 CLN2 clinical rating scale (CLN2 CRS) in patient 1 (bullets) and patient 2 (boxes) during two years of treatment with cerliponase alfa. Closed bullets/boxes represent total sum score of all four domains and open bullets/boxes represent the sum score of the motor and language domain

Patient 2 had maximum scores on all subdomains of the CLN2 CRS at baseline (CLN2 CRS total score: 12), which remained the same over the course of treatment (Fig. 1).

\section{Evaluation of gross motor function}

Patient 1 lost her ability to walk without support at the age of 43 months. At baseline she had severe hypertonia, most prominent in her lower limbs, for which a combination of trihexyphenidyl and baclofen was started. She also received night splints to correct contractures of her feet. This resulted in some improvement of her mobility, where she was able to crawl again. Her Gross Motor Function Measure-66 (GMFM-66) total score at baseline was 46 (95\% CI 43.7-47.8) and decreased to 39 (95\% CI 36.4-41.5) after 2 years of treatment (Fig. 2).

Patient 2 had age-appropriate gross motor skills at baseline (GMFM-66 total score 66 (95\% CI 62.9-68.4) and he continued to have a normal gross motor development thereafter. At the age of 49 months he was still gaining motor skills, while patient 1 at the same age had already lost many of her motor abilities (Fig. 2). His GMFM-66 total score after 2 years of treatment was 84 (95\% CI 78.8-89.3).

\section{Neuropsychological development}

At baseline (age 46 months) patient 1 had, based on the the Bayley Scales of Infant and Toddler Development, 3rd Edition, Dutch version (BSID-III-NL), a cognitivedevelopmental age equivalent to 24 months and receptive and expressive communication skills equivalent to a developmental age of 27 months. After 2 years of therapy (age 72 months) her cognitive-developmental age was equivalent to 11 months and her receptive and expressive

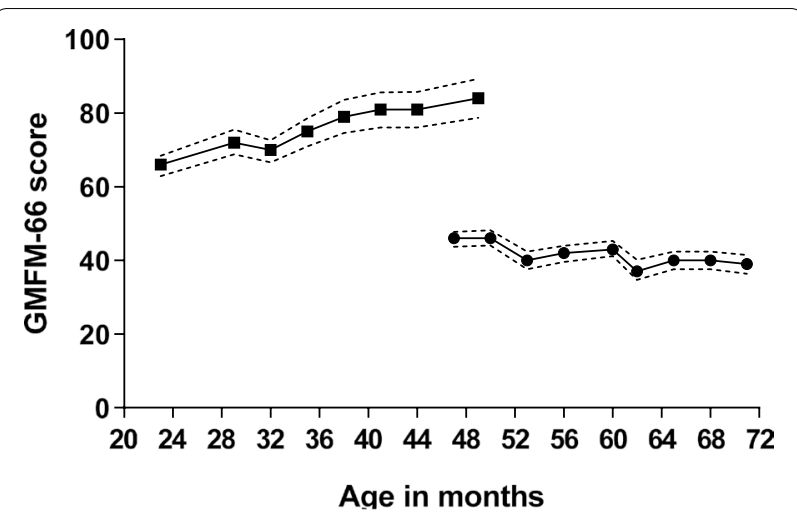

Fig. 2 Gross motor function measure-66 (GMFM-66) total scores in patient 1 (bullets) and patient 2 (boxes) during two years of treatment with cerliponase alfa. Dotted lines represent $95 \%$ confidence intervals

communication skills were equivalent to a developmental age of 10 and 17 months, respectively.

At baseline (age 22 months) patient 2 had age-appropriate scores on both the cognitive and language scales of the BSID-III-NL. At age 29 months his cognitive and communication skills were equivalent to a developmental age of 27 months. At age 48 months, after 2 years of treatment, his cognitive-developmental age was assessed to be 39 months, but we were not able to reliably assess his language skills due to lack of sufficient cooperation during testing. We therefore estimated his language skills according to the BSID-III-NL based on parent reports. His receptive and expressive communication skills seemed equivalent to a developmental age of 39-42 months. He had just started attending a regular primary school.

\section{Visual impairment}

Parents did not notice any visual impairments of both patients during the study. Both patients had a normal ophthalmological examination at baseline and after 2 years of treatment. In patient 1 visual evoked potentials (VEP) performed at baseline and at regular intervals during 18 months of treatment showed giant potentials consistent with CLN2 disease [17]. Due to agitation it was technically impossible to repeat a VEP at 24 months of treatment. Electroretinography (ERG) at 6 months of treatment showed normal latency times, an ERG could not be done at baseline as well as during 6-24 months of treatment due to agitation.

In patient 2 a VEP/ERG was not performed at baseline. Subsequent VEPs showed bilateral prolonged p100 latency with normal amplitude. No giant potentials were seen during 2 years of treatment. ERGs were normal. 


\section{Neuroimaging}

The baseline brain magnetic resonance imaging (MRI) of patient 1 showed mild cerebral and cerebellar atrophy with concomitant ex vacuo dilatation of the ventricle system (Fig. 3), and T2 hyperintense signal abnormalities of the periventricular white matter, consistent with CLN2 disease $[6,17,18]$. The brain MRI after 2 years of treatment showed progressive cerebral and cerebellar atrophy with progressive ex vacuo dilatation of the ventricles (Fig. 3) and mild progression of the periventricular white matter abnormalities.

Patient 2 had a normal brain MRI at baseline. The brain MRI after 2 years of treatment (at age 48 months) showed no signs of cerebral or cerebellar atrophy (Fig. 3), but did show a mild hyper intense T2 signal of the periventricular white matter at the occipital horns, which was considered to be related to terminal myelination zones.

\section{Epilepsy}

At baseline patient 1 exhibited a mixed type of epilepsy with tonic-clonic seizures, myoclonic seizures, atonic seizures and absences. Her anticonvulsants were adapted over time. With the combination of lamotrigine, levatiracetam and clobazam, she had multiple short (duration up to $20 \mathrm{~s}$ ) partial complex seizures (both tonic and atonic) and/or absence seizures per day with only sporadic myoclonic seizures at her last evaluation. The last tonic-clonic grand mal seizure occurred at age 59 months. No aggravation of the seizures was noticed during infusion of cerliponase alfa nor in the days thereafter.

Patient 2 did not have any signs of epilepsy during 2 years of treatment and remains free of epilepsy to date.

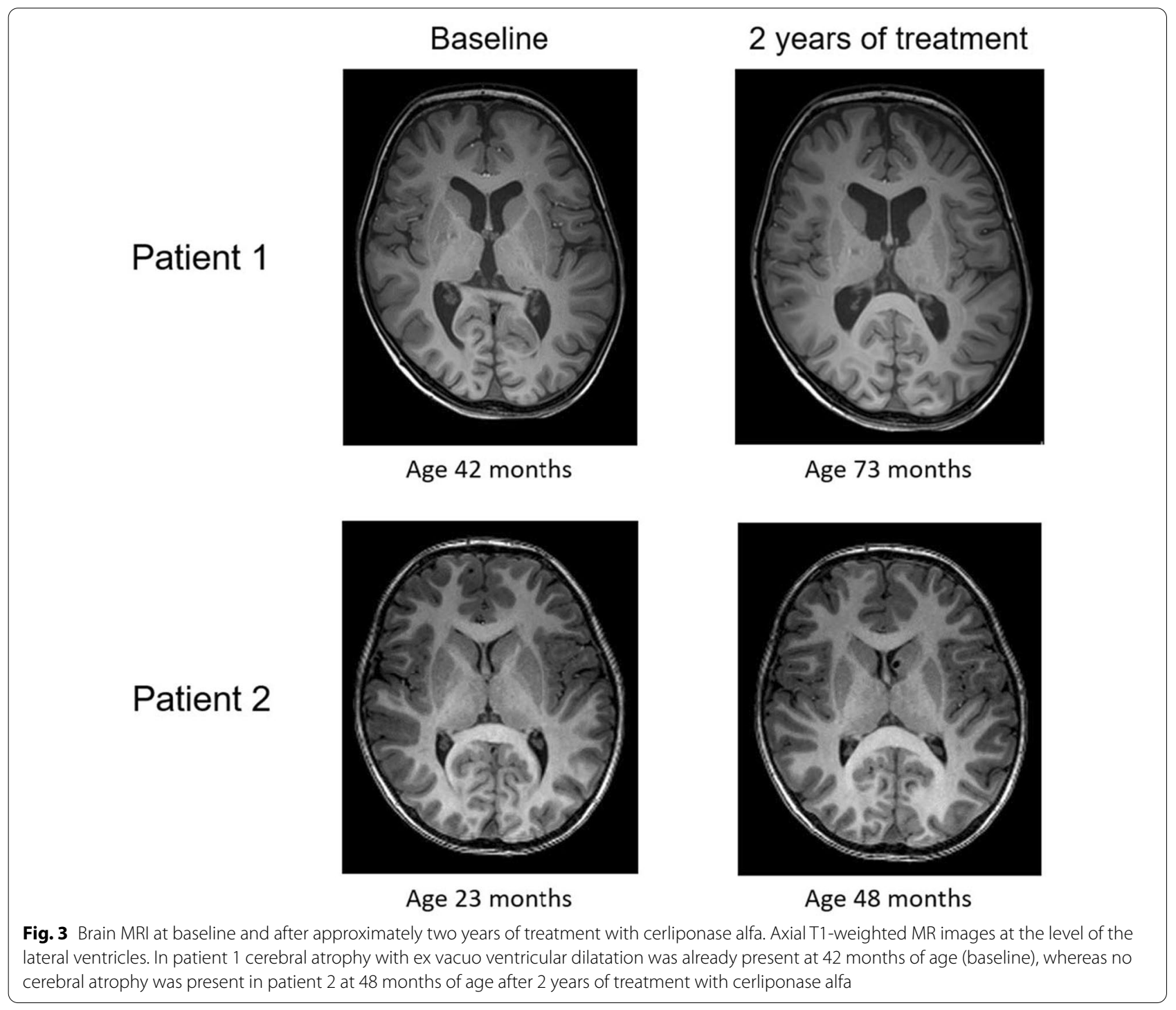




\section{Behavior and sleeping disorders}

Before the start of therapy, the behavior of patient 1 had changed from a happy to a frustrated child, crying often and expressing a lot of anger. She also developed a sleeping disorder waking up in the middle of the night and staying awake thereafter without any additional sleep during the day. Providing structure and sleep hygiene had no effect and different medication combinations, such as melatonin, hydroxyzine, pipamperone and pregabalin, had varying degrees of effect, but did not resolve the sleeping disorder. Her parents reported this as the most important burden of the disease for them.

No behavior or sleeping disorders were reported in patient 2.

\section{Assessment of treatment safety Infusion associated reactions}

Both patients exhibited infusion associated reactions on cerliponase alfa, patient 1 after the 15th infusion and patient 2 after the 6 th infusion. In both patients these reactions were mild (Common Terminology Criteria for Adverse Events (CTCAE) grade 1 or 2) and included development of an exanthema/maculo-papular rash covering less than $30 \%$ of body surface area (CTCAE grade 2), fever up to $39.0^{\circ} \mathrm{C}$ (CTCAE grade 1 ), nausea (CTCAE grade 1 ) and sinus tachycardia (CTCAE grade 1$)$. In both patients these reactions could be well managed by adding corticosteroids (oral dexamethasone $0.15-0.4 \mathrm{mg} /$ $\mathrm{kg})$ to their premedication, with which these reactions did not reoccur. Similar reactions have been observed in patients after cerliponase alfa administration $[15,19]$. No prolonged hospitalization was required. CSF cultures remained negative.

\section{Discussion}

Classic late-infantile CLN2 disease is a rare rapidly progressive neurodegenerative disorder, resulting in early death. Enzyme replacement therapy with cerliponase alfa now makes it possible to treat patients with CLN2 disease. Schulz et al. [15] showed this therapy to be effective in slowing down disease progression. We evaluated treatment outcome after 2 years of treatment with cerliponase alfa in two siblings with CLN2 disease, both heterozygous for the two most common pathogenic TPP1 variants and with the same environmental factors, and found cerliponase alfa not only to be effective in slowing down disease progression, but also in extending the presymptomatic phase of the disease.

Natural history studies have shown a homogeneous course of disease progression in CLN2 disease, reflected in a rapid decline in the combined motor and language score of the CLN2 CRS between age 3-6 years [4]. After start of ERT patient 1 had a slower disease progression than predicted based on natural history. She lost 1 point on the combined motor and language scale after 15 months of treatment with cerliponase alfa due to a regression in use of language. This is in line with the reported results in treated patients $[15,20]$. The initial increase in CLN2 CRS total score was explained by a better control of her epilepsy, but fluctuated over time due to recurring grand mal seizures. This is why the CLN2 CRS combined motor and language score was chosen as the primary outcome measure over the CLN2 CRS total score in the pivotal trial [15], since cerliponase alfa is less likely to influence seizure control, as seizures result from existing neurological damage, or affect loss of vision as the retina will not be reached with intracerebroventricular administration. Her younger brother did not show any decline in score on the CLN2 CRS at age 4 years, where we would have expected him to become symptomatic around age 3-3.5 years [4], also based on symptom onset in his sister.

Although proven of great value in evaluating the clinical course in CLN2 disease and assessing treatment efficacy with cerliponase alfa [5], the CLN2 CRS is a rough scoring tool, in which a decline in score equals a large loss in functionality within that specific domain, and more subtle changes in functionality are not detected. In addition, the score could not capture an improvement in motor skills in patient 2 as he already exhibited the maximum score. We therefore chose to also evaluate the patients using domain specific tests. The GMFM-66 was used to evaluate motor function. This motor assessment tool was developed for children with cerebral palsy, but has been used to assess motor function in other disorders as well [21-24]. Although the symptoms in patients with CLN2 disease meet the criteria for the use of the GMFM-66 [25], no other studies have reported its use in this patient population. The motor impairment in patient 1 was clearly demonstrated in her GMFM-66 total score at baseline. Her score showed a small decline during 2 years of treatment, but was expected to show a more rapid decline based on the homogenous regression in motor function observed in untreated patients [4]. The GMFM-66 total score in patient 2 showed an age-appropriate increase (the maximum total score of 100 can only be reached from age 5 years onward without motor disabilities) during 2 years of treatment and was remarkably higher than that of his sister at the same age (Fig. 2). This clearly demonstrates efficacy of treatment with cerliponase alfa with respect to motor function.

The results from the neuropsychological evaluation were more difficult to interpret as these were influenced by motor function of patient 1 , and lack of sufficient cooperation during testing of both patients. Therefore, it is plausible that they sometimes underperformed during 
testing given what was reported by their parents when the children were at home. Despite this a clear regression in developmental age was seen in patient 1 , which aligned with both the loss in language score on the CLN2 CRS as well as the progression of cerebral and cerebellar atrophy on brain MRI. However, a more rapid cognitive decline was expected based on natural history data [4-6]. In patient 2 an increase in developmental age was seen. Although this was assessed not to be fully age-appropriate, and taking into account some degree of variability which can be seen in clinical phenotype between siblings with the same disorder, his neurocognitive development was much better than his sister at the same age where he was able to start regular primary school. His brain MRI showed mild periventricular signal changes, which can still be age-appropriate (terminal myelination zones) or, alternatively, may be a first sign of CLN2 as this has been described in infantile onset NCL [26], but not specifically in CLN2 patients. Follow-up brain MRI studies will be needed to make this discrimination. No cerebral or cerebellar atrophy was seen in patient 2 after 2 years of treatment, which is in sharp contrast with the brain MRI of his sister at a younger age (Fig. 3).

Cerliponase alfa is not expected to influence the onset of vision loss in CLN2 as it does not reach the retina with intracerebroventricular administration. No apparent visual problems were seen in both patients, although it should be noted that overt visual impairment occurs at a later stage in CLN2 disease [5]. Patient 1 had giant potentials and patient 2 had a prolonged P100 latency time on VEP studies. Both findings have been described in CLN2 patients [17], this could potentially be the first manifestation of CLN2 disease in patient 2.

The therapy was tolerated well by both patients. Although both had mild infusion associated reactions (CTCAE grade 2 at most), in accordance with reported adverse events $[15,16]$, these could be managed well with oral corticosteroids and never interfered with cerliponase alfa infusions.

\section{Conclusions}

In conclusion, we show that intracerebroventricular ERT with cerliponase alfa can delay disease onset in CLN2 disease. In addition, the results in patient 1 confirm that cerliponase alfa slows down disease progression in CLN2 disease. Our and previously reported results [15, 20] demonstrate that intracerebroventricular ERT can have a significant impact on the disease course of CLN2 disease, as well as potentially other neurodegenerative lysosomal storage disorders, which is a breakthrough in the treatment of these severe disorders. Studies with a larger study population and longer follow up duration, like the ongoing phase 2 trial (NCT02678689), are needed to confirm our results and to establish long term treatment efficacy. If disease onset can be delayed for an extended period of time this may pave the road for possible inclusion of CLN2 disease in newborn screening programs and provide a bridge towards the development of innovative treatments, like gene therapy currently under development (NCT01161576; NCT00151216).

\section{Methods}

\section{Treatment with cerliponase alfa}

Both patients received a Rickham intracerebroventricular reservoir before they started treatment with biweekly intracerebroventricular infusions of $300 \mathrm{mg}$ cerliponase alfa $\left(\right.$ Brineura $^{\circledR}$, Biomarin Pharmaceutical Inc.). We adhered to protocols for intracerebroventricular device access and infusion of cerliponase alfa [27, 28]. Antihistamines (clemastine $0.025 \mathrm{mg} / \mathrm{kg}$ ) and paracetamol $(20$ $30 \mathrm{mg} / \mathrm{kg}$ ) were administered as premedication $30 \mathrm{~min}$ before each infusion. During administration, heart rate, oxygen saturation, blood pressure and temperature were monitored. During the first 7 infusions, both patients remained hospitalized overnight. Thereafter, infusions were given in a daycare setting where both patients were observed for an extra three hours after completion of the infusion.

\section{Assessment of treatment efficacy CLN2 clinical rating scale}

The CLN2 Clinical Rating Scale (CLN2 CRS) [5, 15] ("Appendix") was used as the primary outcome measure in order to compare results with those reported in the first patient trial with cerliponase alfa [15]. We compared the sum score of the motor and language domains (max score 6; primary outcome measure of treatment efficacy in the clinical trial), as well as the total score of the four subdomains (max score 12) in both patients. The CLN2 CRS was administered by the same two clinicians $(\mathrm{HHH}$, JMPH) every 3 months.

\section{Evaluation of motor function}

Next to the CLN2 Clinical Rating Scale motor function was evaluated by the same physiotherapist (LJG) every 3 months with the Gross Motor Function Measure-66 (GMFM-66) Dutch version [29]. The GMFM-66 consist of 66 items, divided into five categories (lying and rolling; sitting; crawling and kneeling; standing; walking, running, and jumping). Each item is scored on a four-point Likert scale. The instrument has been validated in children with cerebral palsy from 5 months to 16 years of age. In order to calculate a total score for the GMFM-66 a computer program, the Gross Motor Ability Estimator (GMAE), is needed with which individual item scores can be converted into an interval level total score. A 
5 -year old child without motor disabilities is able to reach the maximum score of 100 . The GMFM-66 is based on the GMFM-88. The advantage of the GMFM-66 over the GMFM-88 is that children who are unwilling or unable to perform certain items can still be scored with an estimation of the scoring on that item based on the child's pattern of responses to other items. This will then result in a GMFM-66 total score with an enlarged confidence interval $[25,30]$.

\section{Neuropsychological outcome}

Neuropsychological outcomes were evaluated at 6-month intervals by the same neuropsychologist (CK) with the Bayley Scales of Infant and Toddler Development, 3rd Edition, Dutch version (BSID-III-NL), as this is the age-appropriate test for children with a developmental age between age 1 and 42 months [31]. The patients were assessed with the cognitive and language scales, which are good predictors of preschool mental test performance. The motor scale was not used as motor function was assessed separately with the GMFM-66.

\section{Neurological outcome}

Epilepsy was evaluated continuously based on parental reports, and was assessed with the CLN2 CRS every 3 months. Anticonvulsant drugs were adjusted accordingly.

An ophthalmological examination was performed at baseline and after 2 years of treatment, to evaluate if there were signs of macular degeneration [32, 33]. Parents were asked about possible visual impairment every three months. As early markers for retinal damage, visual evoked potentials (VEP) and electroretinogram (ERG) were attempted every 6 months.

Both MRI brain studies of patient 1 , as well as the follow-up MRI of patient 2, were performed on a $3 \mathrm{~T}$ MRI scanner. The imaging protocol included a 3D T1-weighted sequence, axial T2-weighted sequence and axial T2 Fluid Attenuated Inversion Recovery (FLAIR) sequence. In addition, Diffusion Weighted Imaging (DWI) and Susceptibility Weighted Imaging (SWI) was performed. No volumetric measurements were done. In patient 1 , the baseline MRI was made at age 42 months and the follow-up MRI at age 73 months. The baseline MRI brain study of patient 2 at age 23 months was acquired on a $1.5 \mathrm{~T}$ MRI scanner using an imaging protocol for neuronavigation purposes only. His follow-up 3 T MRI scan was performed at age 48 months. All MRI studies were done under general anesthesia.

\section{Assessment of treatment safety}

Both patients were monitored during and after treatment with cerliponase alfa (see 'Treatment with cerliponase alfa' section). Data regarding adverse events and any interventions needed to mitigate infusion associated reactions were recorded at every treatment. Adverse events were graded according to the Common Terminology Criteria for Adverse Events (CTCAE) toxicity scale, version 5.0 [34].

To monitor for possible bacterial colonization of the intracerebroventricular device and central nervous system infections, cerebrospinal fluid (CSF) was aspirated before every infusion and sent out for cell count, Gram staining and culturing up to 14 days after aspiration.

\section{Appendix}

CLN2 clinical rating scale

\begin{tabular}{|c|c|c|}
\hline Functional domain & Performance & Score \\
\hline \multirow[t]{4}{*}{ Motor function } & $\begin{array}{l}\text { Has grossly normal gait, no } \\
\text { prominent ataxia, no patho- } \\
\text { logic falls }\end{array}$ & 3 \\
\hline & $\begin{array}{l}\text { Has independent gait as } \\
\text { defined by ability to walk } \\
\text { without support for } 10 \text { steps; } \\
\text { obvious instability and pos- } \\
\text { sibly intermittent falls }\end{array}$ & 2 \\
\hline & $\begin{array}{l}\text { Requires external assistance to } \\
\text { walk or can only crawl }\end{array}$ & 1 \\
\hline & Can no longer walk or crawl & 0 \\
\hline \multirow[t]{4}{*}{ Language } & $\begin{array}{l}\text { Has apparently normal lan- } \\
\text { guage that is intelligible and } \\
\text { grossly age-appropriate, with } \\
\text { no decline noted }\end{array}$ & 3 \\
\hline & $\begin{array}{l}\text { Has language that has recog- } \\
\text { nizable abnormalities but } \\
\text { includes some intelligible } \\
\text { words; may form short sen- } \\
\text { tences to convey concepts, } \\
\text { requests, or needs }\end{array}$ & 2 \\
\hline & $\begin{array}{l}\text { Has language that is hard to } \\
\text { understand with few intel- } \\
\text { ligible words }\end{array}$ & 1 \\
\hline & $\begin{array}{l}\text { Has no intelligible words or } \\
\text { vocalizations }\end{array}$ & 0 \\
\hline \multirow[t]{4}{*}{ Seizures (grand mal) } & $\begin{array}{l}\text { No seizures per 3-month } \\
\text { period }\end{array}$ & 3 \\
\hline & $\begin{array}{l}1 \text { to } 2 \text { seizures per 3-month } \\
\text { period }\end{array}$ & 2 \\
\hline & 1 seizure per month & 1 \\
\hline & $>1$ seizure per month & 0 \\
\hline \multirow[t]{4}{*}{ Visual function } & $\begin{array}{l}\text { Recognizes desirable object, } \\
\text { grabs at it }\end{array}$ & 3 \\
\hline & $\begin{array}{l}\text { Grabbing for objects uncoor- } \\
\text { dinated }\end{array}$ & 2 \\
\hline & Reacts to light & 1 \\
\hline & No reaction to visual stimuli & 0 \\
\hline
\end{tabular}




\section{Abbreviations}

TPP1: Tripeptidyl peptidase; CLN2 CRS: CLN2 clinical rating scale; GMFM-66: Gross motor function measure-66; BSID-III-NL: Bayley Scales of Infant and Toddler Development, 3rd Edition, Dutch; VEP: Visual evoked potentials; ERG: Electroretinogram; MRI: Magnetic resonance imaging; CTCAE: Common terminology criteria for adverse events; CSF: Cerebrospinal fluid; FLAIR: Fluid attenuated inversion recovery; DWl: Diffusion weighted imaging; SWI: Susceptibility weighted imaging.

\section{Acknowledgements}

We would like to thank the parents of the patients described in this paper for allowing us to report on the outcome data of their children.

\section{Authors' contributions}

JS and $\mathrm{HHH}$ : study design, data collection, manuscript concept, drafting of the manuscript, final approval. JMPH, LJG and CK: data collection, study design, critical reading of the manuscript, final approval. SS, MHGD: data collection, critical reading of the manuscript, final approval. EHJ, JKHS, and ATP: critical reading of the manuscript, final approval. All authors read and approved the final manuscript.

\section{Funding}

This study was supported by Stichting Stofwisselkracht (https://www.stofw isselkracht.nl/lopende-onderzoeken/classic-lateinfantile-neuronal-ceroid-lipof uscinosis-cln2-in-the-netherlands-incidence-and-efficacy-of-intracerebroven tricular-enzyme-replacement-ther).

\section{Availability of data and materials}

Data sharing is not applicable to this article as no datasets were generated or analysed during the current study.

\section{Declarations}

\section{Ethics approval and consent to participate}

Parents provided written informed consent for the use of the anonymized data of their children. As these data were obtained within a standardized clinical follow-up protocol formal approval from a medical ethical committee was not required as the study did not fall under the scope of the Dutch Medical Research Involving Human Subjects Act.

\section{Consent for publication}

Parents provided written informed consent for the publication of the anonymized data of their children.

\section{Competing interests}

$\mathrm{HHH}$ has received a speaker fee, a participation fee for an advisory board and reimbursement for the ongoing participation in the 190-504 registry study from BioMarin International Limited under an agreement between BioMarin International Limited and Erasmus MC University Medical Center. BioMarin International Limited was not involved at any stage of the study or during the preparation of the manuscript. All other authors declare no competing interests.

\section{Author details}

'Department of Pediatrics, Center for Lysosomal and Metabolic Diseases, Erasmus MC, University Medical Center Rotterdam, PO Box 2040, 3000 CA Rotterdam, The Netherlands. ${ }^{2}$ Department of Pediatric Physiotherapy, Erasmus MC, University Medical Center Rotterdam, Rotterdam, The Netherlands ${ }^{3}$ Department of Child and Adolescent Psychiatry/Psychology, Erasmus MC, University Medical Center Rotterdam, Rotterdam, The Netherlands. ${ }^{4}$ Department of Clinical Genetics, Erasmus MC, University Medical Center Rotterdam, Rotterdam, The Netherlands. ${ }^{5}$ Department of Radiology and Nuclear Medicine, Erasmus MC, University Medical Center Rotterdam, Rotterdam, The Netherlands. ${ }^{6}$ Department of Pediatric Neurosurgery, Erasmus MC, University Medical Center Rotterdam, Rotterdam, The Netherlands.

Received: 4 March 2021 Accepted: 6 May 2021

Published online: 14 May 2021

\section{References}

1. Sleat DE, Donnelly RJ, Lackland H, Liu CG, Sohar I, Pullarkat RK, et al. Association of mutations in a lysosomal protein with classical late-infantile neuronal ceroid lipofuscinosis. Science. 1997;277(5333):1802-5.

2. Sleat DE, Gin RM, Sohar I, Wisniewski K, Sklower-Brooks S, Pullarkat RK, et al. Mutational analysis of the defective protease in classic late-infantile neuronal ceroid lipofuscinosis, a neurodegenerative lysosomal storage disorder. Am J Hum Genet. 1999;64(6):1511-23.

3. Suopanki J, Partanen S, Ezaki J, Baumann M, Kominami E, Tyynela J. Developmental changes in the expression of neuronal ceroid lipofuscinoseslinked proteins. Mol Genet Metab. 2000;71(1-2):190-4.

4. Nickel M, Simonati A, Jacoby D, Lezius S, Kilian D, Van de Graaf B, et al. Disease characteristics and progression in patients with late-infantile neuronal ceroid lipofuscinosis type 2 (CLN2) disease: an observational cohort study. Lancet Child Adolesc Health. 2018;2(8):582-90.

5. Steinfeld R, Heim P, von Gregory H, Meyer K, Ullrich K, Goebel HH, et al. Late infantile neuronal ceroid lipofuscinosis: quantitative description of the clinical course in patients with CLN2 mutations. Am J Med Genet. 2002;112(4):347-54.

6. Worgall S, Kekatpure MV, Heier L, Ballon D, Dyke JP, Shungu D, et al. Neurological deterioration in late infantile neuronal ceroid lipofuscinosis. Neurology. 2007;69(6):521-35.

7. Dozieres-Puyravel B, Nasser H, Elmaleh-Berges M, Lopez Hernandez E, Gelot A, llea A, et al. Paediatric-onset neuronal ceroid lipofuscinosis: first symptoms and presentation at diagnosis. Dev Med Child Neurol. 2020;62(4):528-30.

8. Kousi M, Lehesjoki AE, Mole SE. Update of the mutation spectrum and clinical correlations of over 360 mutations in eight genes that underlie the neuronal ceroid lipofuscinoses. Hum Mutat. 2012;33(1):42-63.

9. Moore SJ, Buckley DJ, MacMillan A, Marshall HD, Steele L, Ray PN, et al. The clinical and genetic epidemiology of neuronal ceroid lipofuscinosis in Newfoundland. Clin Genet. 2008;74(3):213-22.

10. Di Giacopo R, Cianetti L, Caputo V, La Torraca I, Piemonte F, Ciolfi A, et al. Protracted late infantile ceroid lipofuscinosis due to TPP1 mutations: Clinical, molecular and biochemical characterization in three sibs. J Neurol Sci. 2015;356(1-2):65-71.

11. Gardner E, Bailey M, Schulz A, Aristorena M, Miller N, Mole SE. Mutation update: review of TPP1 gene variants associated with neuronal ceroid lipofuscinosis CLN2 disease. Hum Mutat. 2019;40(11):1924-38.

12. Mole SE, Williams RE, Goebel HH. Correlations between genotype, ultrastructural morphology and clinical phenotype in the neuronal ceroid lipofuscinoses. Neurogenetics. 2005;6(3):107-26.

13. Williams RE, Adams HR, Blohm M, Cohen-Pfeffer JL, de Los RE, Denecke J, et al. Management strategies for CLN2 disease. Pediatr Neurol. 2017;69:102-12.

14. Schulz A, Kohlschutter A, Mink J, Simonati A, Williams R. NCL diseasesclinical perspectives. Biochim Biophys Acta. 2013;1832(11):1801-6.

15. Schulz A, Ajayi T, Specchio N, de Los RE, Gissen P, Ballon D, et al. Study of intraventricular cerliponase alfa for CLN2 disease. N Engl J Med. 2018;378(20):1898-907

16. Markham A. Cerliponase alfa: first global approval. Drugs. 2017;77(11):1247-9.

17. Jadav RH, Sinha S, Yasha TC, Aravinda H, Gayathri N, Rao S, et al. Clinical, electrophysiological, imaging, and ultrastructural description in 68 patients with neuronal ceroid lipofuscinoses and its subtypes. Pediatr Neurol. 2014;50(1):85-95.

18. Johnson AM, Mandelstam S, Andrews I, Boysen K, Yaplito-Lee J, Fietz M, et al. Neuronal ceroid lipofuscinosis type 2: an Australian case series. J Paediatr Child Health. 2020;56(8):1210-8

19. Lewis G, Morrill AM, Conway-Allen SL, Kim B. Review of cerliponase alfa: recombinant human enzyme replacement therapy for late-infantile neuronal ceroid lipofuscinosis type 2. J Child Neurol. 2020;35(5):348-53.

20. Estublier B, Cano A, Hoebeke C, Pichard S, Scavarda D, Desguerre I, et al. Cerliponase alfa changes the natural history of children with neuronal ceroid lipofuscinosis type 2: The first French cohort. European journal of paediatric neurology : EJPN : official journal of the European Paediatric Neurology Society. 2020;30:17-21.

21. Russell DJ, Avery LM, Rosenbaum PL, Raina PS, Walter SD, Palisano RJ. Improved scaling of the gross motor function measure for children with cerebral palsy: evidence of reliability and validity. Phys Ther. 2000:80(9):873-85. 
22. Russell D, Palisano R, Walter S, Rosenbaum P, Gemus M, Gowland C, et al. Evaluating motor function in children with Down syndrome: validity of the GMFM. Dev Med Child Neurol. 1998;40(10):693-701.

23. Sato T, Adachi M, Nakamura K, Zushi M, Goto K, Murakami T, et al. The gross motor function measure is valid for Fukuyama congenital muscular dystrophy. Neuromuscul Disord. 2017;27(1):45-9

24. Nelson L, Owens H, Hynan LS, lannaccone ST, Am SG. The gross motor function measure is a valid and sensitive outcome measure for spinal muscular atrophy. Neuromuscul Disord. 2006:16(6):374-80

25. Russell DJ, Rosenbaum P, Wright M, Avery LM. Gross motor function measure (GMFM-66 \& GMFM-88) users manual. United Kingdom: Mac Keith Press; 2002

26. van der Knaap MS, Bugiani M. Leukodystrophies: a proposed classification system based on pathological changes and pathogenetic mechanisms. Acta Neuropathol. 2017;134(3):351-82.

27. Slavc I, Cohen-Pfeffer JL, Gururangan S, Krauser J, Lim DA, Maldaun M, et al. Best practices for the use of intracerebroventricular drug delivery devices. Mol Genet Metab. 2018;124(3):184-8.

28. Schwering C, Kammler G, Wibbeler E, Christner M, Knobloch JK, Nickel $\mathrm{M}$, et al. Development of the "Hamburg best practice guidelines for ICV-enzyme replacement therapy (ERT) in CLN2 disease" based on 6 years treatment experience in 48 patients. J Child Neurol. 2021:883073821989154

29. Beckers LWME, Bastiaenen CHG. Application of the gross motor function measure-66 (GMFM-66) in Dutch clinical practice: a survey study. BMC Pediatr. 2015;15(1):146.
30. Avery LM, Russell DJ, Raina PS, Walter SD, Rosenbaum PL. Rasch analysis of the Gross Motor Function Measure: validating the assumptions of the Rasch model to create an interval-level measure. Arch Phys Med Rehabil. 2003;84(5):697-705.

31. Steenis LJ, Verhoeven M, Hessen DJ, van Baar AL. Performance of Dutch children on the Bayley III: a comparison study of US and Dutch norms. PLOS ONE. 2015:10(8):e0132871.

32. Orlin A, Sondhi D, Witmer MT, Wessel MM, Mezey JG, Kaminsky SM, et al Spectrum of ocular manifestations in CLN2-associated batten (JanskyBielschowsky) disease correlate with advancing age and deteriorating neurological function. PLOS ONE. 2013;8(8):e73128.

33. Raitta C, Santavuori P. Ophthalmological findings in infantile type of so-called neuronal ceroid lipofuscinosis. Acta Ophthalmol (Copenh). 1973:51(6):755-63.

34. U.S. Department of Health and Human Services. Common Terminology Criteria for Adverse Events (CTCAE). Version 5.0. https://ctep.cancer.gov/ protocoldevelopment/electronic_applications/docs/CTCAE_v5_Quick_ Reference 8.5x11.pdf. Accessed 26 Jan 2021.

\section{Publisher's Note}

Springer Nature remains neutral with regard to jurisdictional claims in published maps and institutional affiliations.
Ready to submit your research? Choose BMC and benefit from:

- fast, convenient online submission

- thorough peer review by experienced researchers in your field

- rapid publication on acceptance

- support for research data, including large and complex data types

- gold Open Access which fosters wider collaboration and increased citations

- maximum visibility for your research: over $100 \mathrm{M}$ website views per year

At $\mathrm{BMC}$, research is always in progress.

Learn more biomedcentral.com/submissions 